\title{
Flexible Productive Systems Modeling and Control Tools: Literature Review
}

\author{
Mazzoni URC*, Asato OL* and Nakamoto FY* \\ * Federal Institute of Education, Science and Technology of São Paulo - Campus São Paulo, São Paulo.
}

\begin{abstract}
The challenges imposed on Manufacturing Systems (MS), given the demands of a dynamic and competitive market, instigates the development of new technologies to promote the reduction of production costs, increase productivity and ensure the level of quality established by the company. Such technologies applied in MS create demands for new paradigms for the design of control systems, mainly about the integration of automated systems, such as multifunction machines, flexible machining centers, intelligent robotic conveyor systems, and the integration of information systems, production planning and management, and manufacturing execution. The main purpose of control system modeling is to represent a real system using conceptual models to visualize, predict and simulate the desired dynamic behavior of the system. This article presents some modeling tools for control systems capable of adequately representing a manufacturing system with all its requirements and intrinsic characteristics, supported by formal methods for structured modeling of the control system.
\end{abstract}

Keywords. Modeling Tools, Productive System Modeling, Petri Nets, E-MFG.

Introduction. The globalization of the market has imposed a particular and previously nonexistent dynamics on MS, which the flexibility of production processes is highly required, with demands for products and services with a gradual reduction of the life cycle, which the rational use of resources is necessary due to the sustainability of industrial processes, quality assurance and reliability as a market differential, a growing volume of data at all levels of the productive system with considerable autonomy of components and equipment. This way, it is necessary to develop new structured models to guarantee the dynamic behavior desirable to the system and effective integration of the automated systems. With the emergence of new technologies and the inherent modernization of industrial parks, it is necessary to develop new models and methodologies to adapt and ensure that the manufacturing system works effectively to produce efficiently. The modeling of the manufacturing control system has been widely studied (1, 2, 3, $5,6,7,19,20,21)$ and aims to represent a real system using conceptual models for visualize, predict and simulate the desired dynamic behavior of the system. The formalism is a factor of extreme importance in the modeling of control system. Overall, formalizing can be defined by describing a process, object, phenomenon, etc., so that anyone can interpret without duality or ambiguity. Formalism ensures a correct interaction of process participants, data consistency, and a more secure process (3). Thus, the main motivation for the research work is the need to adequately represent a manufacturing system with all its intrinsic requirements and 
characteristics supported by formal methods and tools for structured modeling of the manufacturing control system.

Flexible Manufacturing System - FMS. A manufacturing system can be defined as a set of people, machines, equipment, tools and other devices that perform activities with the same objective, transforming an input resource (raw material, material) in a final output product with value added, or the provision of service $(8,9)$. FMS generally consist of autonomous workstations with a high degree of integration. These stations are interconnected by a material handling, transport and storage system. It is used a central computer to control and monitor the various activities that occur in the system, determining the route of the various parts to the appropriate stations by controlling the operations previously programmed in the different stations (8). In FMS a large number of activities occur in parallel and concurrency, sharing finite resources and making it difficult to implement the system when compared to sequential systems.

Control System Modeling. A model, in engineering, can be defined as an abstraction of reality, or a simplified representation of a system with the intention of studying it. To be able to represent a real-world system and draw conclusions about a particular application, a detailed model is necessary. The models should consider the representation of all components involved in the system as entities, attributes, activities, events and states $(9,10)$. As real-world models tend to be complex because of the large number of information, computational tools are often used to run models and simulations. Computational tools revolutionized the industry, provided several benefits such as improved corporate management, increased production and reduced development costs and projects. Therefore they are widely used in industries to achieve high level of global competition. In the present work some tools were studied with the objective of selecting the tools that can be useful for manufacturing control system modeling.

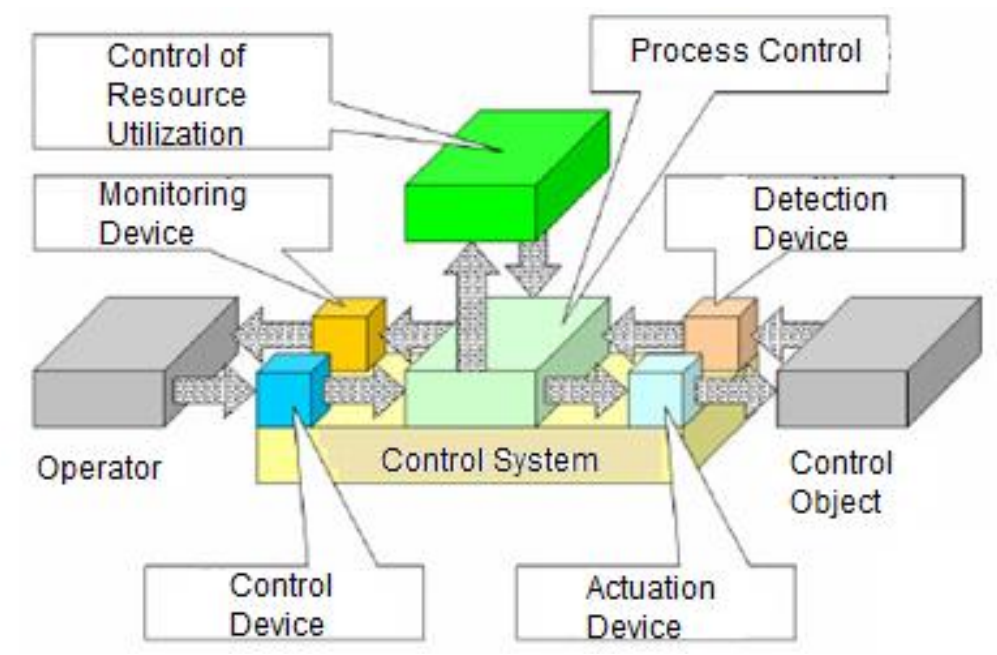

Figure 1: Conceptual model of control system $(9,10)$. 
Unified Modeling Language - UML. The UML is an object-oriented tool for modeling information systems, has a standardized language for modeling, with vocabulary and rules originally focused on the conceptual and physical representation of a software system, which can be used in business modeling and systems in general $(11,12)$. The UML is suitable for modeling systems, which can include corporate information systems to be distributed to web-based applications and even real-time embedded complex systems. The current version 2.5 of the UML has fifteen diagrams divided into two categories: Structural (seven diagrams) and Behavioral (eight diagrams). Figure 1 illustrates the division of UML diagrams.

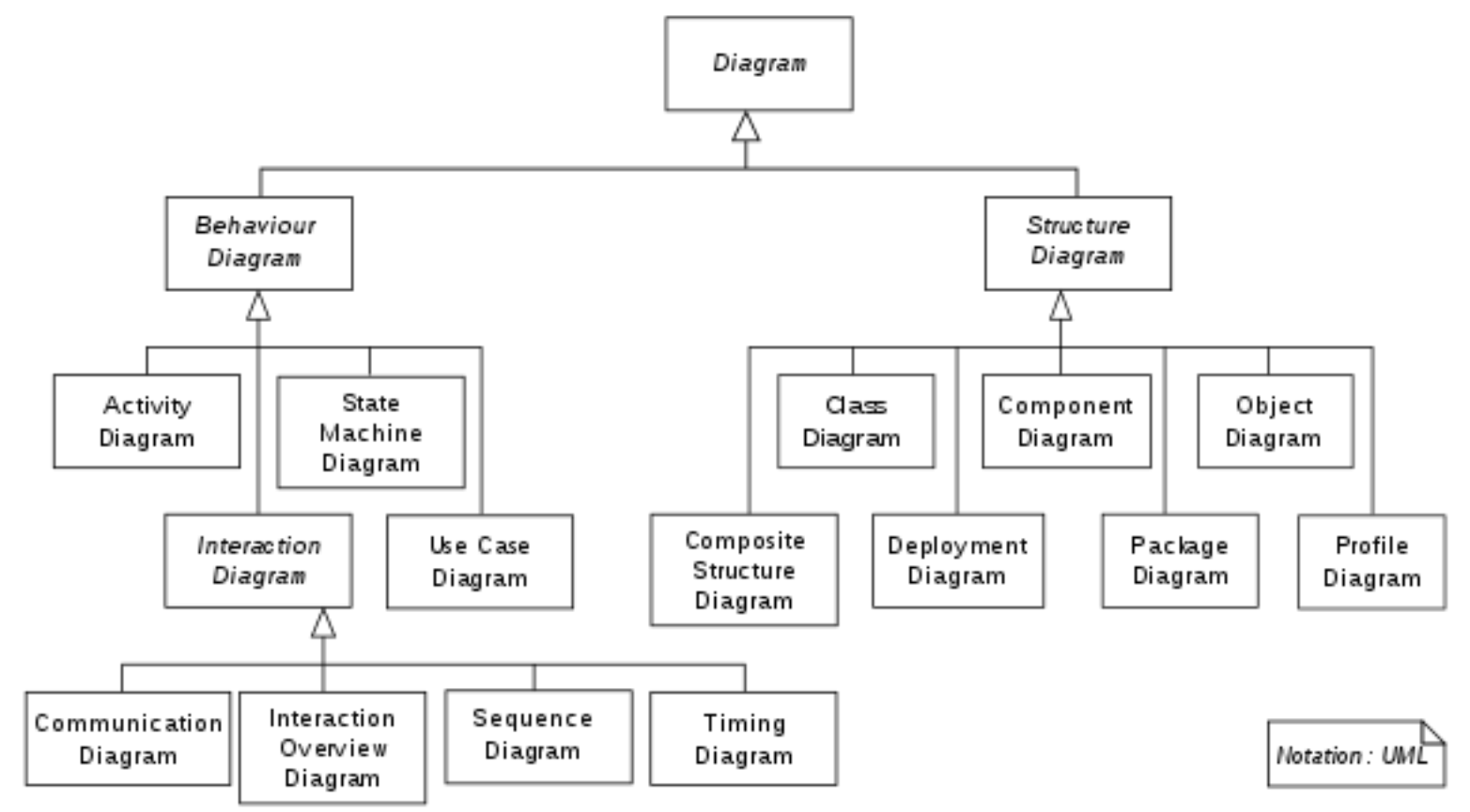

Figure 2: UML diagrams (12).

Structural diagrams are related to the structural aspect both from the point of view of the system and of the classes. They exist to visualize, specify, construct and document the static aspects of a system, in other words, the representation of its skeleton and structures (11). Behavior diagrams show the dynamic behavior of objects in a system, including its methods, collaborations, activities, and states. The dynamic behavior of a system can be described as a whole host of changes that occur over time. The behavior diagrams provide a high-level abstraction for the system's functionalities (12).

Petri Nets - PN. It is the result of Carl Adam Petri's 1962 thesis; the thesis presented the PN as a mathematical formalism to describe the relationships between conditions and events in asynchronous systems in computers and communication protocols. The PN is a graphical and 
mathematical tool that adapts well to a large number of applications such as: evaluation of performance, analysis and formal verification in discrete systems, communication protocols, control of manufacturing workshops, design of real-time and/or distributed software, information systems, transportation system, logistics, database management, interface man-machine and multimedia (14). The PN as a graphical and mathematical representation technique provides a uniform environment for modeling, formal analysis and systems design to Discrete Event System (DES) (15). Overall, a PN is a directed bipartite graph composed by place (represented graphically by a circle or ellipse), transition (represented by a rectangle or bar) and oriented arcs connecting the places with transitions and vice versa. The arcs can have a weight to assign a characteristic to the marking as shown in the figure 2 (a). The dynamics of a system is represented by the marking concept that associates to each place a set of marks (represented graphically by points) that indicate the current state of the system and the concept of the transition trigger that establishes rules for the evolution of the marking. The mark dynamic evolution of a PN is defined by the number of tags and their distribution in the graph, which is changed with the PN transitions trigger, as shown in the figure 2 (b). When a transition fires, it removes the marks from the places of entry (precondition) and creates new marks at the exit places (postcondition).

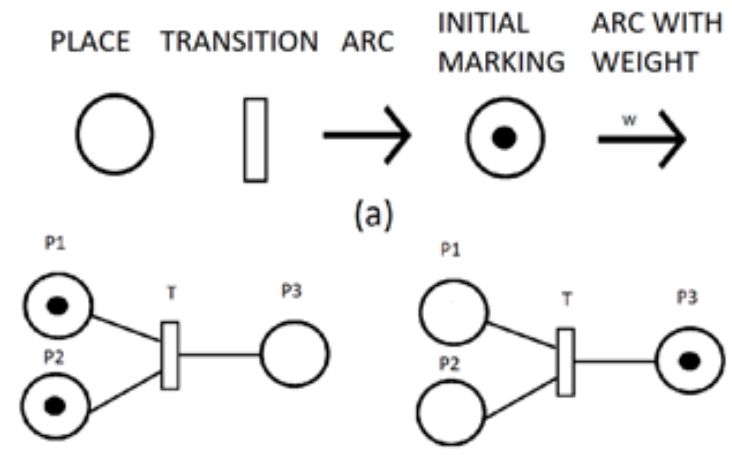

(b)

Figure 3: (a) Graphical representation of the elements of the PN, (b) Transition trigger dynamics.

Enhanced Mark Flow Graph- E-MFG. The MFG (Mark Flow Graph) modeling tool is an interpreted net derived from Petri Nets $(13,16,17)$ developed for the modeling and control of systems. The MFG, in addition to representing the dynamics of the system's operation, allows the representation of external elements as control signals and also allows the dynamic representation of situations such as: asynchrony, parallelism, competition and interlocking (1). The E-MFG modeling tool includes the individual marks and structural elements of the MFG (Mark Flow Graph) (18) and allows the manipulation of tags with attributes without, however, escaping from the conventional elementary net model. It has the ability to model and control changes in brand information and selection of tasks associated with boxes $(9,21)$. In E-MFG the marks are accompanied by a vector of attributes that guarantee their individuality. To these attributes can 
be associated diverse information regarding the product, the process and the control. The zero attribute means absence of these attributes (2).

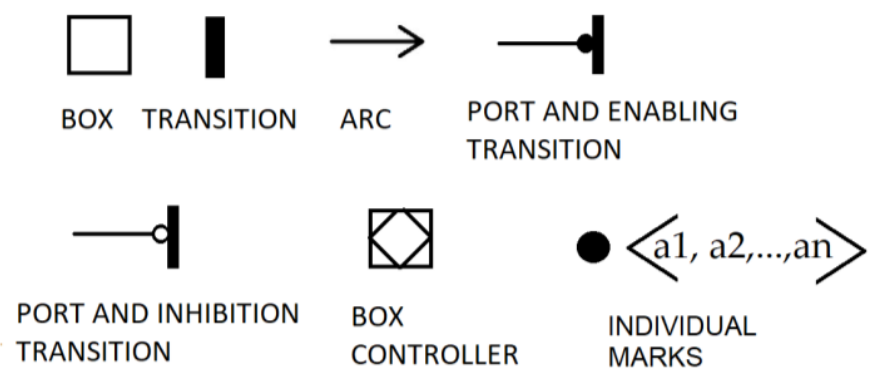

Figure 4: E-MFG basic elements.

Production Flow Schema - PFS. It is a tool that allows constructing a conceptual model at a relatively high level of abstraction without considering the dynamics of the system $(2,18)$. The PFS has three basic elements as shown in the figure 4: active element or activity (they are elements that carry out the action); passive element or distributor (they are elements that present the state in which the object that undergoes the action is) and oriented arcs (they are elements that associate activities to the distributor and vice versa). The PFS takes advantage of the fact that systems can be modeled with a set of activities, which perform a process or a function in the items, by a sequence of activities and distribution steps that, in turn, perform the flow items through the system or process. The modeling procedure in PFS is based on applying the technique of successive refinement, in order to gradually and naturally insert the detail of the process in the model. The PFS, unlike the PNs, do not have markup and it attends the characteristics of DESs as: sequence, parallelism, synchronization, competition and resource sharing, as shown in the figure 4.

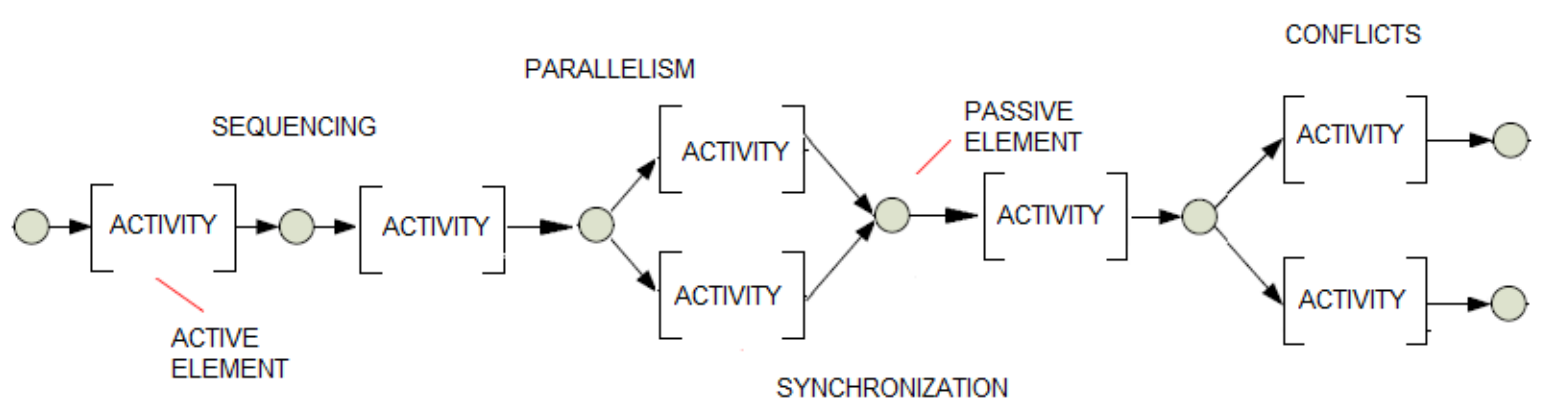

Figure 5: Structural elements of PFS adapted (5). 
PFS/E-MFG Methodology. The PFS/E-MFG methodology is based on the methodologies of top-down project and on the hierarchical structured approach through successive refinement (1). The activities and distributors of the high-level model in PFS are replaced by a detailed network in PFS or E-MFG, and in the more detailed level of the project, the net must have only E-MFG models to be implemented. The use of this methodology allows a conceptual model with natural language of easy understanding, such as PFS, to become a formal model developed in E-MFG at the lower levels.

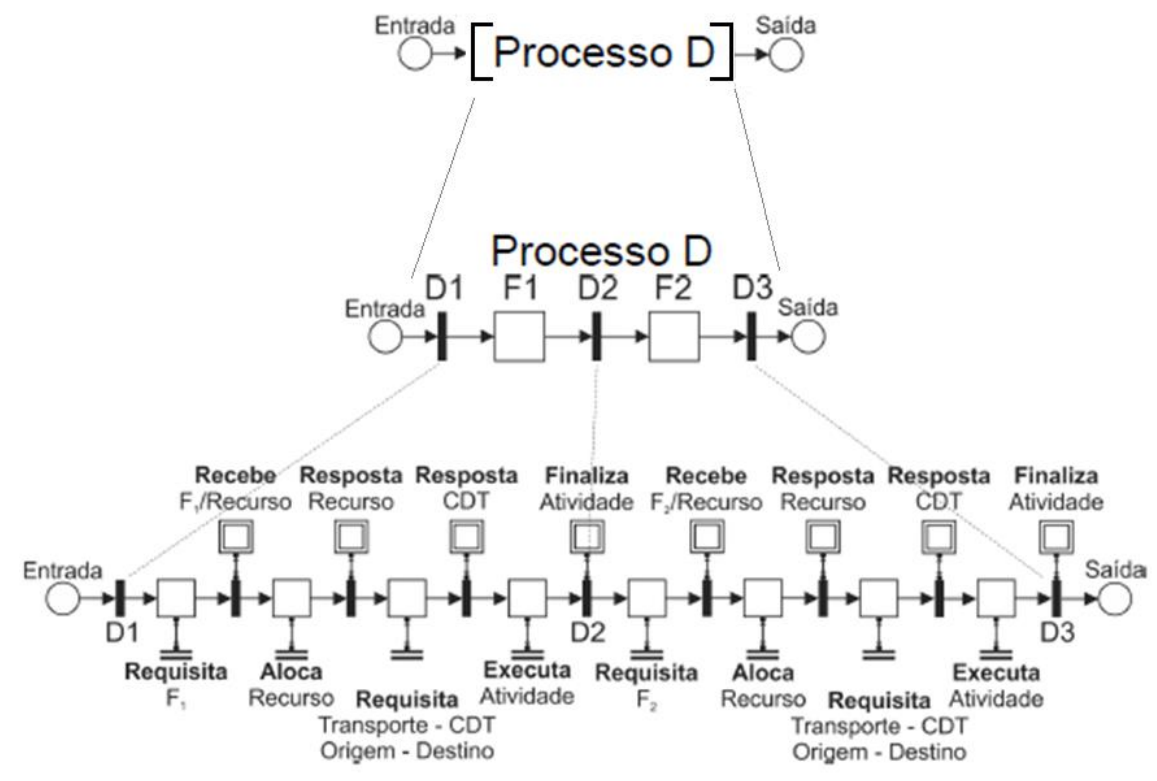

Figure 6: Methodology PFS/E-MFG (2).

\begin{tabular}{|l|}
\hline \multicolumn{1}{|c|}{ part } \\
\hline attribute \\
\hline method \\
\hline
\end{tabular}

\begin{tabular}{|l|}
\hline \multicolumn{1}{|c|}{ process } \\
\hline attribute \\
\hline method \\
\hline
\end{tabular}

\begin{tabular}{|l|}
\hline \multicolumn{1}{|c|}{ resource } \\
\hline attribute \\
\hline method \\
\hline
\end{tabular}

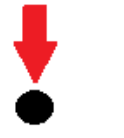

Data Structure in UML
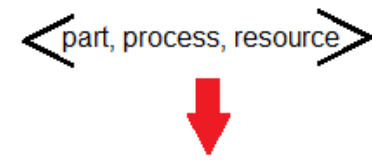

Production Flow Schema
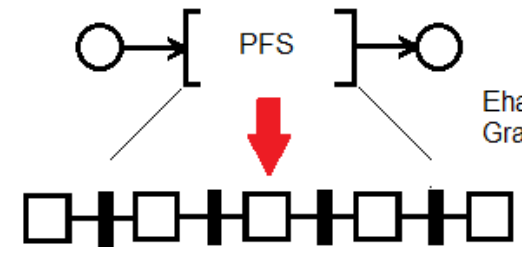

Figure 7: Application example of modeling tools. 
Conclusion. In the present work, some control system modeling tools were studied with the objective of selecting the tools that can be useful for modeling FMS. To perform the requirements survey and the interaction between the elements of the control system, the tool Unified Modeling Language is going to be used (11), what offers a standardized graphical language that allows the visualization, specification, construction and documentation of the elements that compose a manufacturing system in the step of surveying specifications and requirements. Considering the research project, the next step will be the improvement of the modeling tool of the UML data structure to the PFS / E-MFG methodology.

Acknowledgments. The authors thank CAPES, IFSP, UNILA and PTI for their support.

\section{References.}

(1) Miyagi PE. Programmable Control- Fundamentals of systems control to discrete events. São Paulo: Ed. Edgard Bluncher Ltda, 1996.

(2) Santos Filho DJ. Design aspects of productive systems. Thesis [free teaching]-Escola Politécnica da Universidade de São Paulo, São Paulo, 2000.

(3) Villani E. Modeling and analysis of supervisory systems. Thesis [PhD]- Escola politécnica da universidade de São Paulo. São Paulo, 2004.

(4) Matsusaki CTM. Distributed control systems modeling and collaborative production systems. Thesis [Phd]- Escola Politécnica da Universidade de São Paulo, São Paulo, 2004.

(5) Morales RAG, Melo JIG, Miyagi PE. Diagnosis and treatment of faults in productive systems based on Bayesian networks and Petri Net. IEEE International conference on automation science and engineering, 2007, Case 2007, pp. 357-362, 2007.

(6) Nakamoto FY, Santos Filho DJ, Miyagi PE. Resource allocation control in flexible manufacturing systems using the deadlock avoidance method. ABCM Series in Mechatronics, pp. 454-460, 2008.

(7) Asato OL, Pessoa MAO, Junqueira F, Santos Filho DJ, Okamoto Junior J, Miyagi PE. Using the enhanced - mark flow graph for dynamic resource allocation in distributed manufacturing. International Journal of Computer Integrated Manufacturing, Vol. 29, pp. 1-14, 2015.

(8) Groover MP. Automation, production systems, and computer-integrated manufacturing. New Jersey: Ed. Prentice-Hall, 2011. pp. 43-60.

(9) Santos Filho DJ, Nakamoto FY, Junqueira F, Miyagi PE. Task control of intelligent transportation vehicles in manufacturing systems. Mechatronics Series 1: Intelligent transportation vehicles, Vol. 1, Dubai: Bentham Science Publishers, pp. 146-169, 2011.

(10) Nakamoto FY. Modular control system design for production systems. Thesis (doctoral)Escola Politécnica da Universidade de São Paulo, São Paulo, 2008.

(11) Booch G, Rumbaugh J, Jacobson I. The unified modeling language user guide, 1999.

(12) OMG. OMG Unified modeling language. Version 2.5 (2015).

(13) Murata T. Petri Nets: Properties, analysis and applications. Proceedings of the IEEE, pp. 541-580, 1989.

(14) Cardoso J, Valette R. Petri Nets. Florianópolis: Editora da UFSC, 212p, 
(15) Zhou M, Zurawski R. Petri nets and industrial applications: A Tutorial. IEEE Transactions on Industrial Electronics, Vol. 41, n. 6, pp. 567-583, 1994.

(16) Peterson JL. Petri Net Theory and the modeling of systems. Englewood Cliffs, N.J., PrinceHall, 1981.

(17) Reising W. Petri nets: An introduction. Springer-Verlag, Berlin Heidelberg, 1985.

(18) Hasegawa K, Takahashi K, Masuda R. Ohno H. Proposal of mark flow graph for discrete System control. Transactions of the society of instrument and control engineers, Vol. 20, n.2, pp.122-129, 1984.

(19) Kubo, R.H., Asato, O.L., Dos Santos, G.A., Nakamoto, F.Y. Modeling of allocation control system of multifunctional resources for manufacturing systems. 12th IEEE International Conference on Industry Applications, INDUSCON 2016. https:// 10.1109/INDUSCON.2016.7874596

(20) Guirro, D.N., Asato, O.L., Santos, G.A dos e Nakamoto, F.Y. Manufacturing operational management modeling using interpreted Petri nets. Gest. Prod., São Carlos, v. 27, n. 2, e3920, 2020. https://doi.org/10.1590/0104-530x3920-20

(21) Nakamoto, F.Y., Miyagi, P.E., Santos Filho, D.J. dos. Automatic generation of control solution for resource allocation using Petri net model. Prod., São Paulo, v. 19, n. 1, p. 8-26, Apr. 2009. http://dx.doi.org/10.1590/S0103-65132009000100002 